\section{GARANTIA DE CALIDAD EN LA OBRA CIVIL DE UN TANQUE PARA GNL ESPAÑA}

\author{
Luis Benito Quintana, \\ Ingeniero de Caminos \\ Director de la División de \\ Control de Obra de INTEMAC
}

$581-29$

\section{SINOPSIS}

Las características especiales de la construcción del TK 2001 para GNL llevaron a utilizar como servicio de control de calidad el Control de Calidad de Producción del Constructor, incorporando la participación de una Agencia de Inspección Independiente para efectuar actividades de Garantía de Calidad y supervisión del mismo.

Se exponen en el presente artículo las actividades desarrolladas por esta organización, prestando especial atención a las relaciones de la misma con el Servicio de Control de Calidad y con la obra.

En la fase previa a la construcción del tanque TK2001 para GNL en la Zona Franca de Barcelona, la entidad propietaria ENAGAS, S. A., mantuvo contactos con distintas empresas relacionadas con el proyecto, construcción y control de calidad de obras de este tipo. Dada nuestra especialización profesional nos correspondió intervenir en los temas relacionados con este último aspecto.

Si bien prácticamente ninguna obra ingenieril puede considerarse como repetición en serie de otra anterior, es evidente que la que nos ocupa, por su naturaleza, dimensiones e incorporación de tecnologia de vanguardia, responde mejor a las características de un prototipo desde este punto de vista.

Por ello las conversaciones mantenidas y actuaciones derivadas entre las distintas partes intere- sadas en la obra: propiedad, proyecto, dirección técnica, construcción, explotación y los organismos especializados asesores han tenido un carácter interactivo en el que por lo que a nosotros respecta han cubierto, en ocasiones, tanto los aspectos de garantía de calidad como los de control de calidad.

\section{Actuaciones en fase de proyecto}

Durante la fase de preparación y puesta a punto del proyecto la Organización de Control de Calidad intervino, fundamentalmente, en la revisión de las especificaciones del mismo.

Esta revisión se llevó a cabo desde el punto de vista de poder desarrollar durante la construcción un Programa de Control de Calidad coherente con la realidad de la misma y el objetivo perseguido.

En este sentido, y dado que en el Proyecto se contemplaba la incorporación de materiales y técnicas innovadoras, o al menos no frecuentes (servidas en algunos casos bajo forma de patentes), se prestó especial atención a distinguir:

a) Materiales que habrian de llegar a la obra con documentación de Control de Calidad suficiente.

b) Materiales cuya aceptación se produciria mediante inspecciones $y$ ensayos realizados en la obra.

c) Unidades de obra que por su tecnologia exigían procedimientos especiales de inspección.

d) Unidades de obra cuyo control podria desarrollarse con los criterios habituales en obra civil.

Se cuidó especialmente mantener un carácter práctico en las especificaciones; así, por ejemplo, aun para materiales tipo a), que debian venir sancionados en origen, se impuso la condición de suministrar un número adicional de unidades con las que, en caso de considerarlo necesario, se podrian haber realizado nuevos ensayos o contraensayos en Laboratorios Especializados. La utilización de estas unidades en el programa de investigación que se desarrolló paralelamente a la construcción de la obra vino a confirmar (al margen incluso de no haberse suscitado problemas de calidad) lo adecuado de la previsión. 


\section{Control de Calidad y Garantia de Calidad}

Consecuencia de las actuaciones desarrolladas en la fase de proyecto fue la propuesta a la entidad propietaria de la obra de un detallado Plan de Control de Calidad que desarrollaba las especificaciones incluidas en el proyecto.

En esos momentos, y estando ya prácticamente decidida la solución constructiva (que llevaria a una Agrupación Temporal de empresas para la construcción) se valoró la incidencia que la aportación de tecnologias propias y el carácter innovador de algunas de ellas habria de tener durante la fase de construcción.

Se consideró que la empresa constructora tendría que desarrollar, como propio Control de Calidad de Producción un programa mucho más extenso e intenso que el normal en una obra civil y que el programa de Control de Calidad de Recepción duplicaria, en cierto modo, algunos de sus puntos (inspecciones y ensayos).

Por ello, con vistas a la construcción, se introdujeron determinadas cláusulas en el contrato en orden a disponer en obra de un Laboratorio y personal especificamente dedicado al control de calidad dentro de la organización del Constructor y se estableció un concurso para designar una Agencia de Inspección Independiente que desarrollaría actividades de asesoramiento en control de calidad y supervisaría, realizando los oportunos contrastes, las actuaciones en este campo del constructor.

Indicaremos además que este servicio de Garantía de Calidad fue adjudicado a dos organizaciones, una, el Instituto Técnico de Materiales y Construcciones (INTEMAC) para la obra civil, y otra, el Centro Nacional de Investigaciones Metalúrgicas (CENIM) para los aspectos relacionados con la tecnologia de las soldaduras y estructura metálica. La presencia de algunas unidades, como los forros metálicos, que junto a especificaciones muy rigurosas en cuanto a las uniones soldadas debian cumplir las correspondientes a encofrado de la pared de hormigón, ponen de manifiesto la dificultad de separar los campos de actuación en algunos casos; indicaremos que ninguna de las organizaciones $y$, en general, ninguna de las entidades que intervinieron en la obra, regateó esfuerzos para el eficaz desempeño de su misión y facilitar el correspondiente de las demás.

\section{Organización del servicio}

Teniendo en cuenta las premisas anteriores, el servicio de Garantia de Calidad se organizó con arreglo al esquema que recoge el gráfico $\mathrm{N} .^{\circ} 1$.

La organización está dimensionada tanto para prestar asesoramiento en cuestiones relacionadas

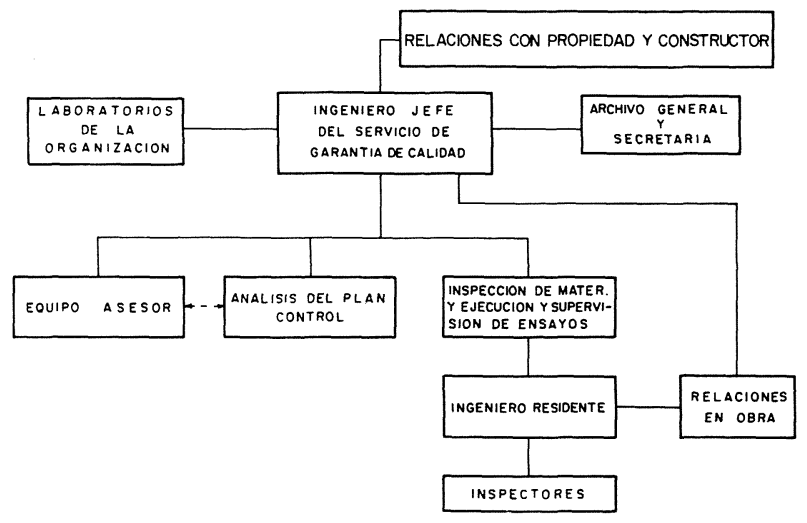

G-1 Organigrama del servicio de garantía de calidad.

con el control de calidad y los resultados obtenidos en el curso de la obra como para la realización de inspecciones y ensayos directos y de contraste con los realizados por el equipo del Constructor.

El esquema se mantuvo a lo largo de toda la obra sin más variación que el número de laborantes presentes en la misma o la intensidad de apoyo de otros laboratorios de la misma Organización, pero ajenos a la obra.

\section{Actuaciones previas a la construcción}

Destacamos a continuación las actuaciones más importantes desarrolladas con el carácter de Garantía de Calidad previamente a la iniciación de la fase constructiva pero ya en contacto con la organización de Control de Calidad del Constructor:

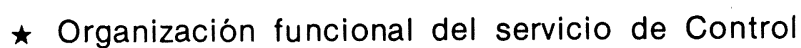
de Calidad y relaciones con el de Garantía de Calidad.

^ Equipo necesario para el Laboratorio de Obra.

* Criterios para el control de entrada de materiales.

* Criterios para la organización de lotes de control de materiales.

^ Actas de toma de muestras; criterios para etiquetado e identificación de muestras.

* Definición de impresos básicos para el registro de actuaciones de control de calidad.

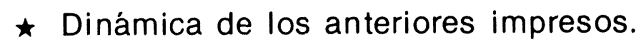

Estos criterios y decisiones se discutieron y tomaron en reuniones conjuntas con la entidad propietaria, Dirección Facultativa, Empresa Constructora y Organizaciones de Control Independientes, para garantizar la necesaria coordinación. 


\section{Actuaciones durante la construcción}

Durante el desarrollo del proceso constructivo las actividades de control de calidad y, por consiguiente, las de garantía de calidad, entran en una fase de actuaciones sistemáticas de acuerdo con el plan de control correspondiente.

Entre estas actuaciones sistemáticas, en orden a reseñar las que tienen más importancia por el volumen de tiempo asignado a las mismas, destacamos las siguientes:

a) Supervisión del control de entrada de materiales (gráfico 2).

b) Formalización de la toma de muestras para control (Actas de toma de muestras).

c) Control de los expedientes de ensayo realizados fuera de la obra (gráfico 3 ).

d) Realización de ensayo de contraste con los anteriores.

e) Control de tajo previo al hormigonado (gráfico 4).

f) Supervisión del control durante el hormigonado (gráfico 5)

\section{OBRA CONTROL DE CALIDAD GARANTIA DE CALIDAD}

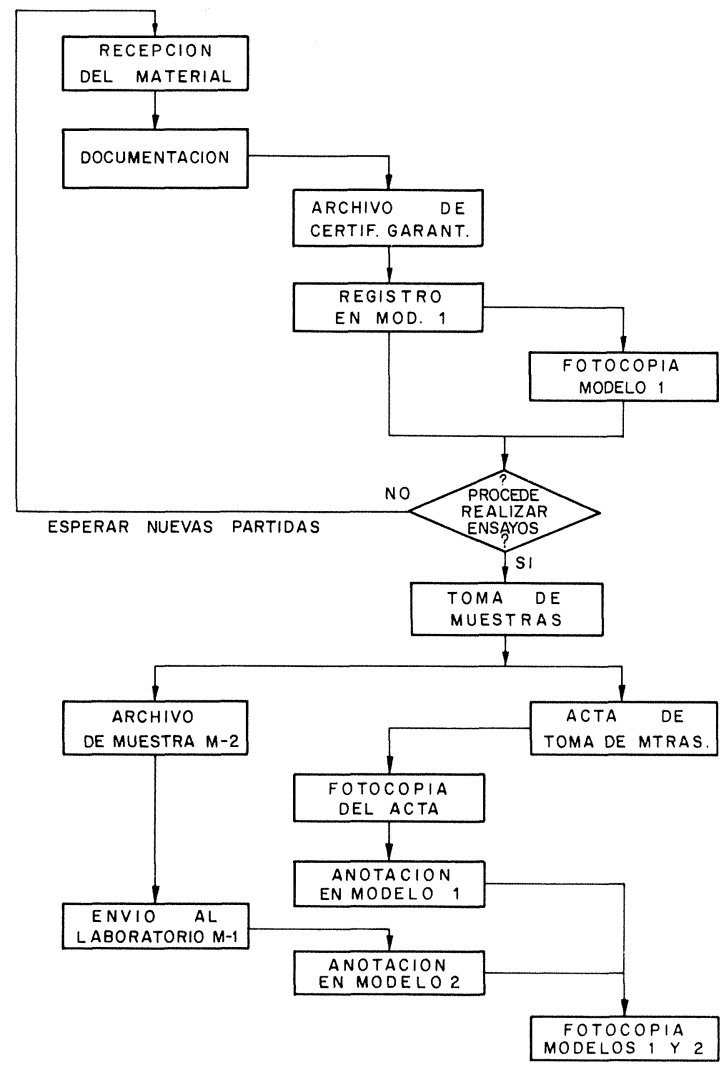

G.2 Diagrama para recepción y control de materiales.

\section{LABORATORIO CONTROL DE CALIDAD GARANTIA DE CALIDAD}

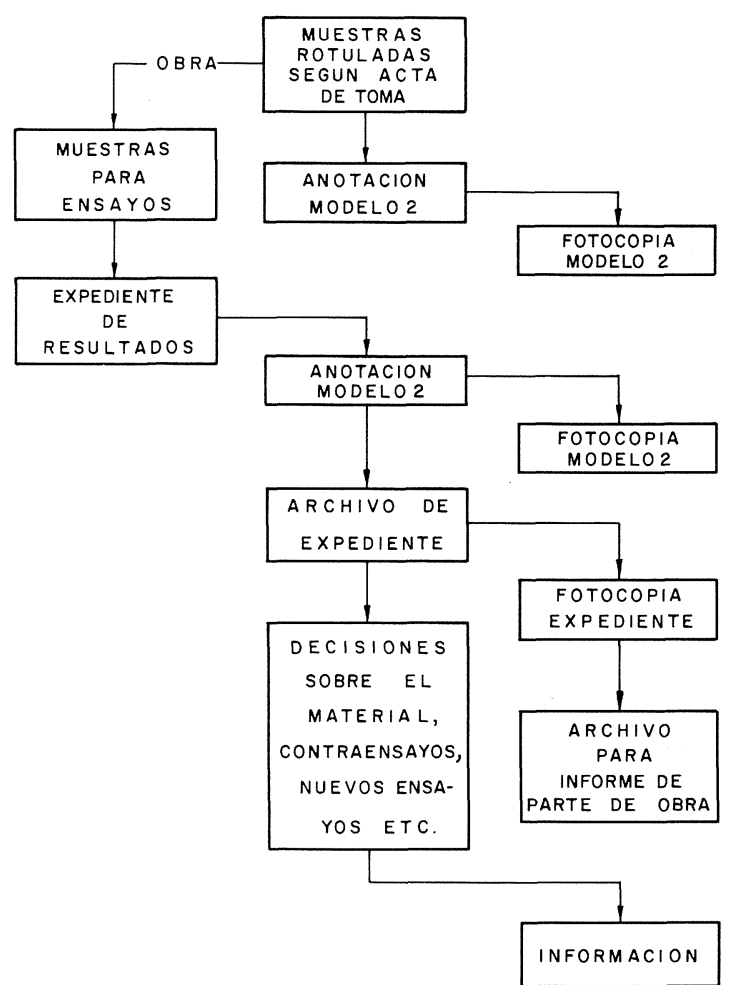

G-3 Diagrama para el control de ensayos realizados fuera del ámíito de la oúra.

g) Supervisión del control mediante probetas moldeadas (gráfico 6)

h) Realización de tomas de contraste de probetas moldeadas.

La realización de tomas de contraste de probetas de hormigón moldeadas se habia previsto en un orden del $5 \%$ de las confeccionadas en la obra. Esta cantidad se incrementó notablemente en determinadas fases de la misma, para aumentar el control de zonas especificas y para atender al Programa de Investigación de Ensayos Criogénicos. Para este último se confeccionaron probetas de $7,5 \times 15$, estudiando su correlación con las normales de $15 \times 30$, y probetas de diseño especial.

Como actividades que tuvieron una importancia localizada en determinada etapa de la obra destacaremos las siguientes:

a) Programación de dosificaciones para desarrollar los ensayos característicos de la instalación.

b) Pruebas de puesta en marcha de la instalación de hormigonado.

c) Tarado periódico de las básculas y dosificadores de la misma. 
OBRA CONTROL DE CALIDAD GARANTIA DE CALIDAD

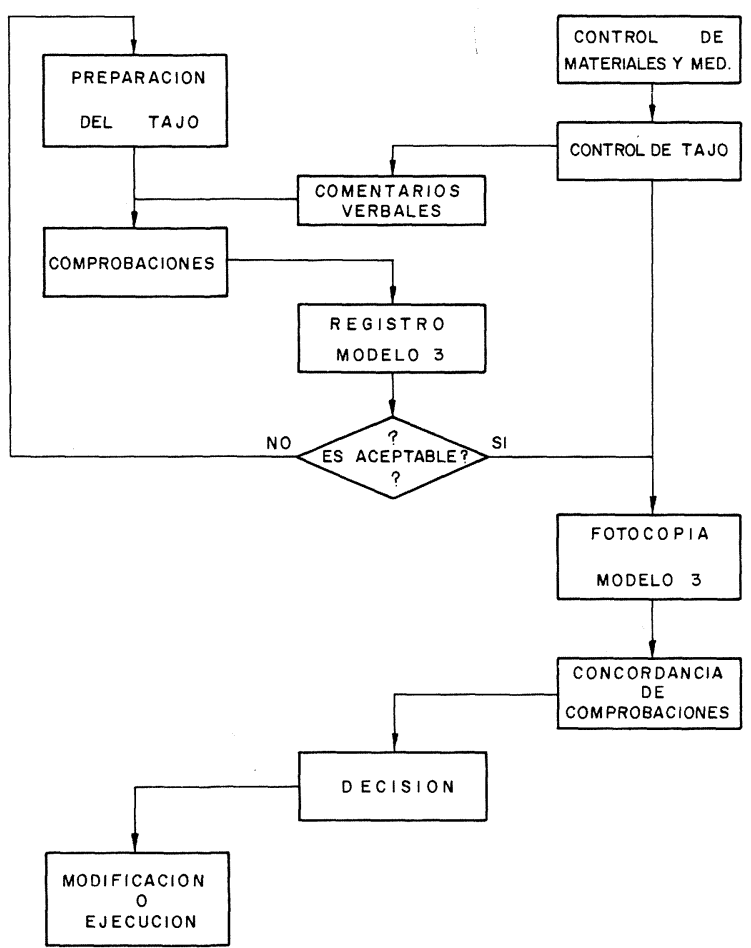

G-4 Diagrama de actuaciones previas al hormigonado de un tajo.

d) Tarado de la prensa del laboratorio de obra.

e) Operaciones de pretensado vertical y relleno de conductos con inyección.

f) Gunitado del muro externo.

g) Operaciones de pretensado horizontal (control de diámetro y tensión en los alambres).

Las actuaciones de garantía de calidad, por tener que desarrollarse sobre actividades realizadas por otros equipos, suelen llevar consigo un incremento en el número de documentos a manejar; en este caso se ha procurado reducir éstos a los indispensables y a establecer los circuitos, tanto informativos como operativos, utilizando fotocopias del documento original con las sucesivas actualizaciones.

Creemos que los gráficos 2 a 6 , correspondientes a la parte de mayor volumen de estas actividades, son suficientemente ilustrativos; en ellos se citan únicamente siete modelos (que no incluimos para no alargar la parte gráfica de estos comentarios) y que son los siguientes:

Modelo 1: Registro de entrada de materiales.

Modelo 2: Registro de ensayos en laboratorios ajenos a la obra.
Modelo 3: Registro de inspecciones de ejecución (por zona de-obra a hormigonar de una vez).

Modelo 4: Parte de control de amasadas producidas en la instalación.

Modelo 5: Parte de control de hormigonado de un tajo.

Modelo 6: Parte de confección y ensayo de probetas de hormigón.

Modelo 7: Cálculo de la resistencia característica estimada de obra para un lote.

\section{Otras actividades}

Aunque son muchas las actividades que en una obra de esta importancia y duración podrian reseñarse, queremos destacar únicamente que a cargo de la Organización de Control Independiente ha corrido el mantenimiento de:

* Archivo de los planos y especificaciones utilizadas en la construcción.

* Archivo de los resultados de las inspecciones y ensayos de ejecución efectuados en la obra.

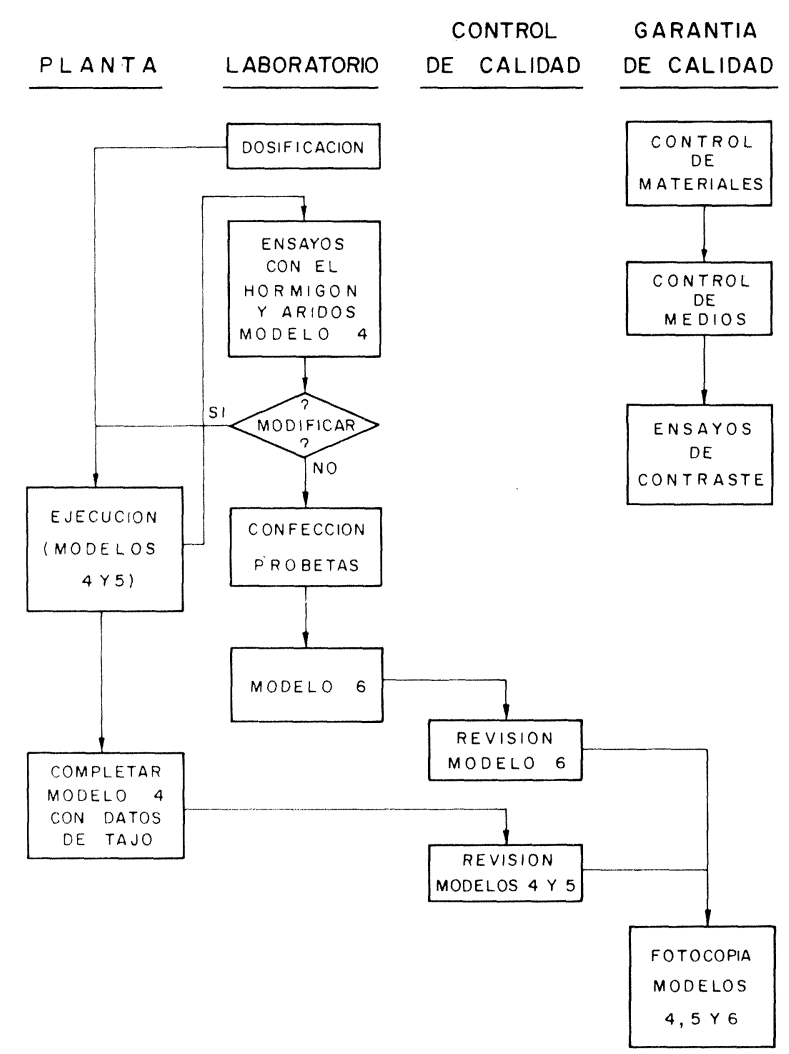

G-5 Diagrama de actuaciones durante el hormigonado de un tajo. 
* Archivo de una copia de los expedientes de ensayos realizados fuera de la obra.

$\star$ Confección de informes mensuales sobre el desarrollo e incidencias habidos en control de calidad.

* Confección de informes de recopilación por partes de obra.

Toda esta documentación fue entregada al Departamento de Proyectos de la entidad propietaria al terminar la obra, de acuerdo con las condiciones del contrato para el desarrollo del servicio.

\section{LABORATORIO CONTROL DE CALIDAD GARANTIA DE CALIDAD}

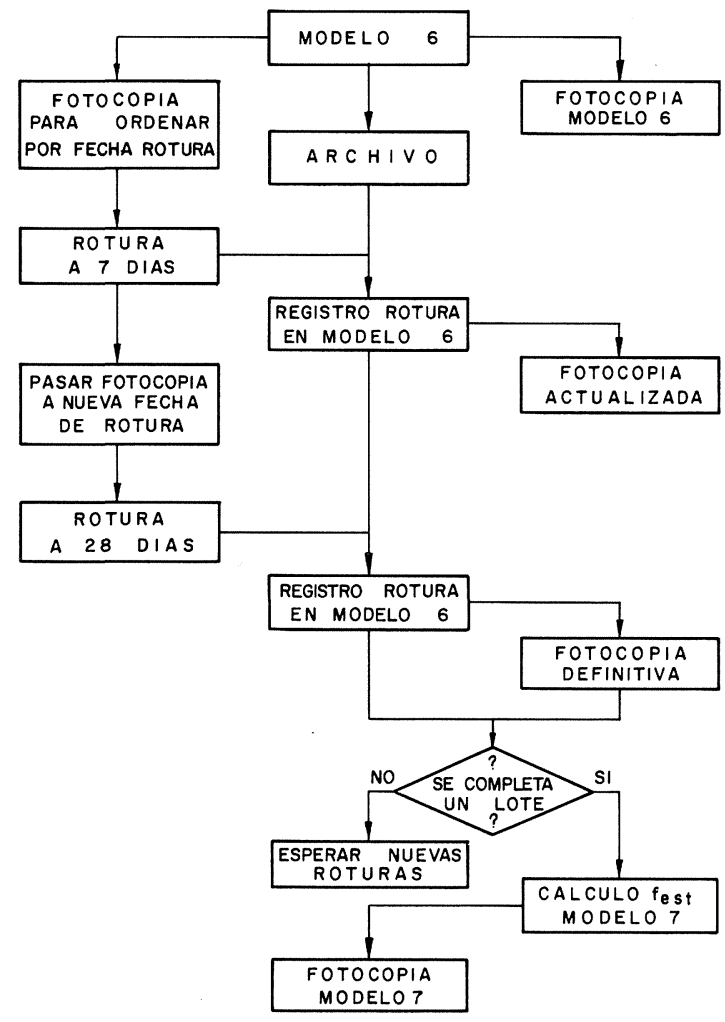

\section{Conclusiones}

La experiencia recogida en la ejecución de esta obra pone de manifiesto la viabilidad de una organización como la descrita.
La extracción de conclusiones de la misma resultaria unilateral por nuestra parte y correspondería hacerla a la Dirección Técnica o, en último extremo, a la entidad propietaria.

El servicio de Control de Calidad del Constructor tiene, en cualquier obra, unos objetivos propios, lógicos, lícitos y necesarios, que no tienen porqué englobar todos los objetivos del Control de Calidad de Recepción.

La presencia de una organización independiente asignada en este caso a misiones de Garantía de Calidad, ha permitido (salvando con la buena voluntad de todos las incursiones y solapes de los campos estrictamente teóricos de actuación) cubrir las necesidades correspondientes al Control de Calidad de Recepción con la flexibilidad exigible a este servicio.

Otro aspecto en el que la presencia de una organización independiente al frente del control de calidad de recepción resulta francamente ventajoso es el correspondiente a la asistencia técnica para la valoración de resultados, toma de decisiones en caso de calidades defectuosas, ampliación del programa inicialmente previsto, etc. Creemos que si, en definitiva, van a mantenerse dos organizaciones dedicadas a actividades de control de calidad, atribuidas en forma simplista al constructor y a la entidad propietaria, seria preferible que los campos de interés y actuación respectivos correspondiesen también a esta misma división $y$, en resumen, fuese una agencia de inspección independiente la que realizase y administrase el Control de Calidad de Recepción y Asistencia Técnica para estas cuestiones.

No queremos terminar sin agradecer a las distintas personas y empresas que intervinieron en la obra y muy particularmente a la empresa propietaria (ENAGAS, S. A.), constructora (Agrupación Temporal PRELOAD-AUXINI) y agencia de inspección para estructura metálica (CENIM) la competencia y dedicación de las personas que intervinieron en los trabajos, sus acertadas observaciones y clima de buen entendimiento para mantener la coordinación necesaria y, finalmente, las facilidades dadas para la redacción de este artículo. 